\title{
EXPERT-SYSTEM BASED MEDICAL STROKE PREVENTION
}

\author{
${ }^{1}$ Anindito, ${ }^{2}$ Bens Pardamean, ${ }^{2}$ Robby Christian and ${ }^{3}$ Bahtiar Saleh Abbas \\ ${ }^{1}$ School of Information Systems, \\ ${ }^{2}$ Graduate Program in Information Systems Management, \\ ${ }^{3}$ Department of Industrial Engineering, \\ Bina Nusantara University, JI. Kebon Jeruk Raya No. 27 Jakarta 11530, Indonesia
}

Received 2013-05-07, Revised 2013-06-07; Accepted 2013-07-10

\begin{abstract}
Stroke always comes unexpected and the general public is not usually aware of its symptoms. Individuals who have had their first stroke with permanent damage could become an economic burden to their family and a social burden to the society due to their unproductive nature. Stroke could be prevented and its risk factors have been identified. Through a stroke prevention information system, the user could be made more aware of stroke risks and symptoms. An expert system would be able to direct and motivate users to keep themselves healthy therefore preventing first and recurrent strokes. The expert system is built using an inference engine that provides stroke risk level based on information provided by the user. Information collected are self measured blood pressure, cigarettes consumed, amount of physical activity and body mass index. Users are presented with suggested preventive tasks to reduce their stroke risk.
\end{abstract}

Keywords: Stroke Prevention, Expert System, Personal Decision Support System

\section{INTRODUCTION}

Medical stroke is the most common cause of death in Indonesia (Sagita, 2013). It amounted to $15.4 \%$ of total deaths which roughly calculated to one person among seven. The stroke prevalence in 2007 is 800 occurrences every hundred thousand citizens. This is comparatively high compared to US at 3.4 every hundred thousand citizens, Singapore at 55 every hundred thousand citizens and Thailand at 11 every hundred thousand citizens.

A stroke is caused by the interruption of the blood supply to the brain, usually because a blood vessel bursts or is blocked by a clot. This cuts off the supply of oxygen and nutrients, causing damage to the brain tissue. Several stroke risk factors have been found such as age, gender, physical activity, hypertension, diabetes, smoking and alcohol consumption (Biswas et al., 2009). As an example (Lynch et al., 2001) found that African
Americans are more prone to stroke than non-African Americans. Controlled stroke risk factors will reduce occurrences.

The (ASA, 2012) noted that by knowledge of personal stroke risk is a beginning step in preventing stroke. While there are uncontrollable variables such as age, gender, race, family medical history and previous stroke history, it is important to notice that there are controllable variables. These variables could be recorded in a database to improve our understanding of stroke studies (Reker et al., 2005). Even professional therapists may not be aware of stroke prevention guidelines and instead focuses on personal knowledge of stroke (Schmid et al., 2008). Hankey and Warlow (1999) calculated that costs to prevent stroke are significantly less than to treat one.

This research proposed to use artificial intelligence, more specifically expert systems in building an Corresponding Author: Anindito, School of Information Systems Bina Nusantara University, JI. Kebon Jeruk Raya No.27 Jakarta 11530, Indonesia 
information system for stroke prevention based on known controllable risk factors.

\subsection{Artificial Intelligence and Expert Systems in Healthcare}

Artificial intelligence involves using the science and engineering of making intelligent machines, especially intelligent computer programs. It is related to the similar task of using computers to understand human intelligence, but AI does not have to confine itself to methods that are biologically observable (McCarthy, 2007a).

Duval and Main (1994) stated that expert systems are a branch of Artificial Intelligence (AI). In building expert systems a knowledge engineer will interview known experts in that domain and tries to embody that knowledge into the system. The usefulness of current expert systems depends on their users having common sense (McCarthy, 2007b). Expert systems are not necessarily high cost according to (Stockdale and Wood, 1992). Kumar and Mishra (2010) highlighted the current trend in using web-based expert systems.

There have been numerous developments in usage of expert systems for healthcare. Zarandi et al. (2010) designed an expert system for asthma diagnosis. Shibata et al. (1998) designed an expert system-based for health promotion of the elderly which is extended by a design from (Iliffe et al., 2005). Panniers (2002) mentioned of developing an expert system for neonatal intensive care. Martin and Harrison (1993) designed an expert system for controlling healthcare costs.

\subsection{Previous Researches on Stroke Scoring and Risks}

Scientists in the past have devised several methods to predict the short-term risk of stroke having had a transient ischaemic attack (Johnston et al., 2007). Ebell (2008) designed a simple scoring system to determine prognosis for people who have had acute stroke. Scoring systems based on discriminant analysis have also been developed such as the Allen score and the Siriraj stroke score (Weir et al., 1994). While these stroke scoring systems have been useful, it is rather different than the purpose of this research which is to reduce stroke risk factors based on known variables.

Boysen and Truelsen (2000) researched several factors that are commonly thought as being associated with stroke risks. Lifestyle factors such as smoking, alcohol, lack of physical activity, cholesterol levels, homocysteine levels and estrogen levels are not found to be directly related to recurrent stroke risks. In the other hand comorbidities such as hypertension, diabetes and atrial fibrillation is found to have direct impact on recurrent stroke risks. Although from this research it might seem that making substantial changes in lifestyle is not important, it is important to note that most of the biologic mechanisms by which different factors influence risk of a first time stroke are also likely to have effect on risk of recurrent stroke. However pharmacological therapy and life modifications are recommended for all patients who have had an ischaemic stroke or transient ischaemic attack (Bushnell and Colon-Emeric, 2009).

\subsection{System Development}

There are three stages in building an expert system (Iliffe et al., 2005). The first stage is building a knowledge base as a reference for stroke risk factors. Controllable variables are measured and standardized. Uniform terminology is also important during the development of expert systems (NHBPEP, 2004). The second stage is building the inference engine where it will match facts with the knowledge domain of the knowledge base to obtain knowledge of stroke risk levels of the user. The third and last stage is the conclusion which is the output from the expert system containing prevention information and other suggestions to decrease stroke risk factors.

\subsection{Building Knowledge Bases}

Knowledge base data is obtained from literature study in stroke prevention. Designed knowledge bases include blood pressure, body mass index, cigarette consumption and physical activity. These knowledge bases are selected due to the ease of gain. Several controllable variables not included are cholesterol levels, blood sugar levels, homocysteine levels and estrogen levels which data cannot be easily obtained in daily activity.

\subsection{Blood Pressure}

Knowledge base for blood pressure is based on systolic blood pressure range (pressure when heart contracts) and diastolic blood pressure range (pressure when heart is filled with blood). Stroke risk increases twice every 20/10 $\mathrm{mmHg}$ (Feigin, 2004).

\subsection{Body Mass Index}

Body Mass Index (BMI) is a simple index of weightfor-height that is commonly used to classify underweight, overweight and obesity in adults (NHBPEP, 2004). It is defined as the weight in kilograms divided by the square of the height in metres $\left(\mathrm{kg} \mathrm{m}^{-2}\right)$. 


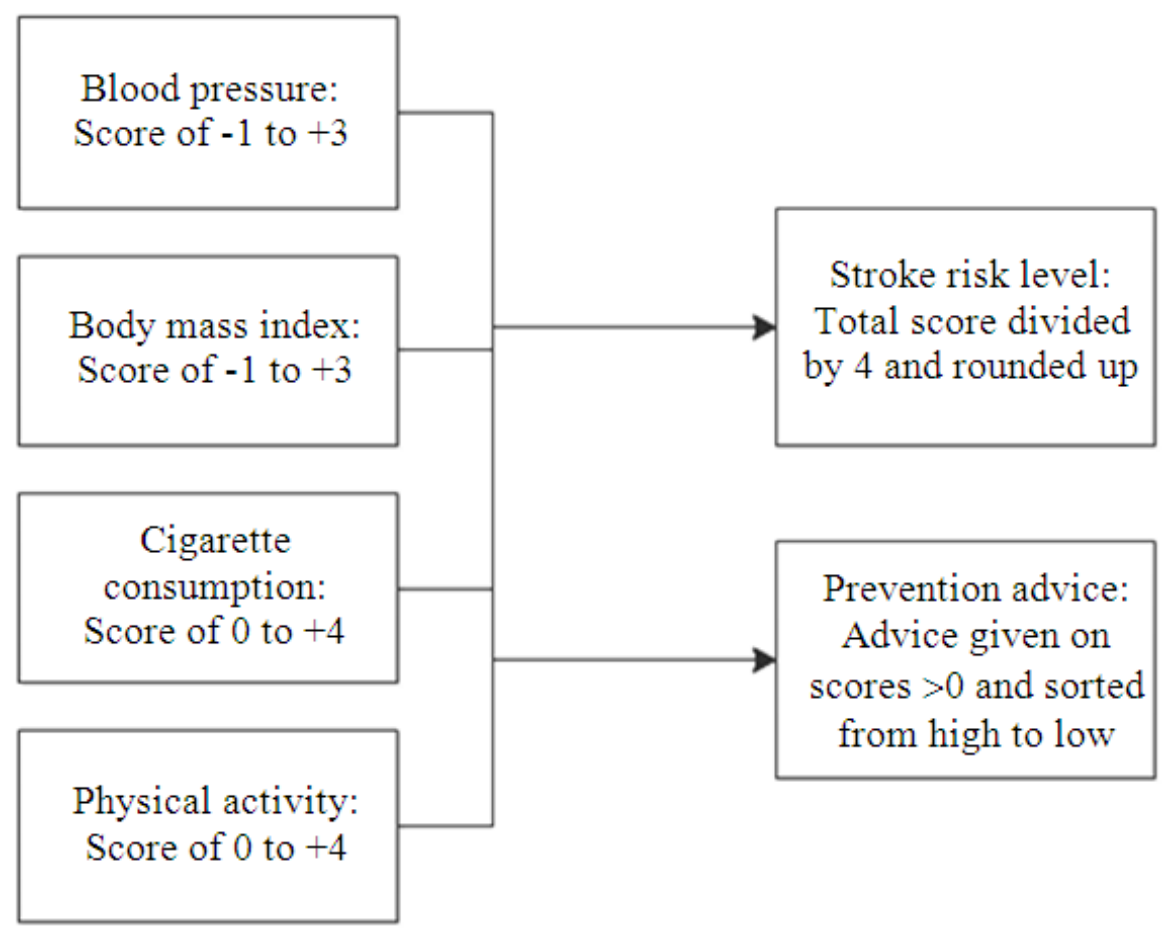

Fig. 1. Inference process of the system

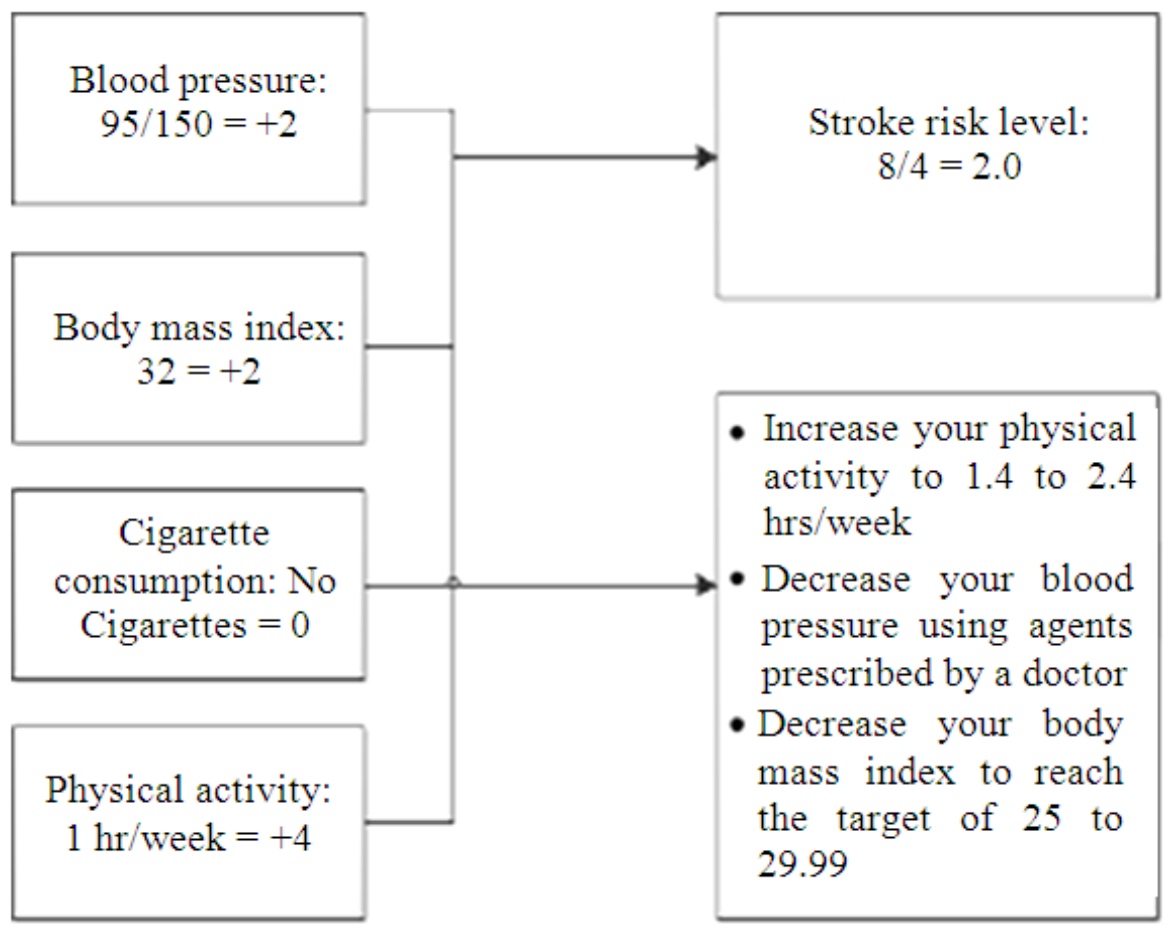

Fig. 2. Sample case for designed stroke prevention system 


\subsection{Cigarette Consumption}

On consumption of cigarettes, the pattern is divided into several categories. While there have been no references on correlation of the number of cigarettes and stroke risk, it is assumed that the target is no consumption. Advice is given based on the current level of consumption. The next target would be a lower level consumption than before and advice is subsequently given until no consumption is achieved.

\subsection{Physical Activity}

Sacco et al. (1998) recommends $3.5 \mathrm{~h}$ per week of moderate activity to reduce stroke risks. This is equivalent of 0 .

\subsection{Inference Engine}

The inference engine is a process in an expert system that matches facts with knowledge contained in the knowledge base to obtain a conclusion in the domain of the expert system. The inference engine in this research is based upon built knowledge bases. A score of +1 is given to a stage increase compared to recommended baseline levels for reducing stroke risks and a score of-1is given to a lower stage below baseline levels.

These scores from available knowledge bases are averaged to obtain a score of stroke risk. Prevention advice is given to the highest score in a knowledge base followed by the next highest. Figure 1 below describes the inference process of the system.

\subsection{Prevention Advice}

Prevention advice given to high blood pressure is to reduce blood pressure levels by using medication or other recommended treatment from their physicians. Prevention advice for body mass index is to lower their body mass index to a stage below current level. Prevention advice for cigarette consumption is based on lowering consumption to a stage lower than current level. Physical activity advice is given to increase physical activity amount in the next stage before current level.

Table 1 shows the knowledge base for blood pressure. Body mass index knowledge base is shown in Table 2. Knowledge base for cigarette consumption is shown in Table $\mathbf{3}$ and knowledge base for physical activity is shown in Table 4. The main target of the system is to reach 0 in stroke risk level. A sample case is illustrated in Fig. 2.
Table 1. Knowledge base for blood pressure (adapted from (NHBPEP, 2004))

\begin{tabular}{llrr}
\hline Code & Classification & Systolic range & Diastolic range \\
\hline H1 & Low & $<90.000$ & $<59.00$ \\
H2 & Normal & $90-120$ & $60-80$ \\
H3 & Prehypertension & $121-139$ & $80-89$ \\
H4 & Stage 1 hypertension & $140-159$ & $90-99$ \\
H5 & Stage 2 hypertension & $\geq 160.000$ & $\geq 100.00$ \\
\hline
\end{tabular}

Table 2. The International Classification of body mass index adapted from (WHO, 2006)

\begin{tabular}{llr}
\hline Code & Classification & Cut-off points \\
\hline B1 & Underweight & $<18.50 .00$ \\
B2 & Normal range & $18.50-24.99$ \\
B3 & Overweight & $25.00-29.99$ \\
B4 & Obese I & $30.00-34.99$ \\
B5 & Obese II & $\geq 35.00 .00$ \\
\hline
\end{tabular}

Table 3. The Classification of cigarette consumption

\begin{tabular}{llr}
\hline Code & Classification & Cigarettes/day \\
\hline S1 & No consumption & 0.00 \\
S2 & Low consumption & $1-60$ \\
S3 & Moderate consumption & $7-12$ \\
S4 & High consumption & $13-24$ \\
S5 & Heavy consumption & $\geq 24.00$ \\
\hline
\end{tabular}

Table 4. The Classification of physical activity

\begin{tabular}{llc}
\hline Code & Classification & Hours/week \\
\hline P1 & Recommended & $>3.5 .000$ \\
P2 & Medium activity & $2.4-3.4$ \\
P3 & Low-medium & $>1.4-2.4$ \\
P4 & Low activity & $>0-1.4 .0$ \\
P5 & No activity & 0.0000 \\
\hline
\end{tabular}

In the sample case it is shown that the user has had no cigarettes and this is aligned with the goal of the system therefore no advice is given.

\subsection{User Interface}

A normal user would open the system in the morning and input data. They would take a blood pressure count and measure their height and weight. Figure 3a below shows the designed input screen for the system.

Textboxes are filled with values from the previous input which is meant as a reminder for users. After the user confirms their input, the system would present the stroke risk levels (Fig. 3b). Stroke risk levels are visualized with a bar to indicate progress of reducing stroke risks. Prevention advice is given from the highest risk factor identified to the lowest. Physical activity data is calculated for 7 days and a daily stroke risk log is kept to track progress. 
Please input your data below for today:

Blood pressure:

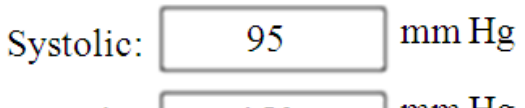

Diastolic: $150 \mathrm{~mm} \mathrm{Hg}$

Body mass index:

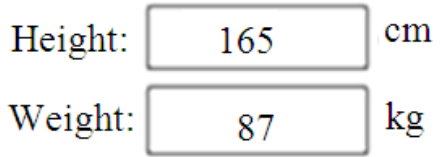

Cigarettes smoked: Pcs

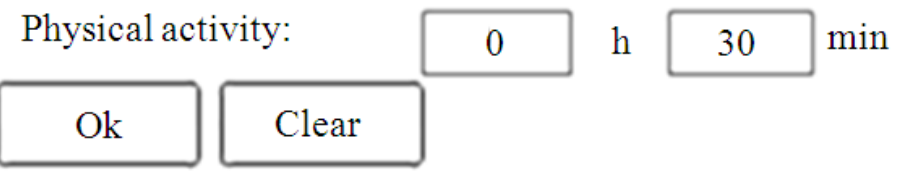

(a)

Stroke prevention system

Your stroke risk level is currently:

\begin{tabular}{|l|l|l|l|l|}
\hline 0 & 1 & 2 & 3 & 4 \\
\hline
\end{tabular}

We suggest that:

- Increase your physical activity to 1.4 to $2.4 \mathrm{~h} /$ week

- Decrease your blood pressure using agents prescribed by a doctor

- Decrease your body mass index to reach the target of 25 to 29.99

(b)

Fig. 3. (a) System interface, (b) Stroke risk levels screen 


\section{CONCLUSION}

We present a simple system to give prevention advice for users in avoiding stroke. The system is designed for simple users with limited equipment availability, in other words not in a medical facility. Equipments needed would only be blood pressure taker, a height measure and a scale to measure weight. System is web-based and can be applied to mobile phones making it portable. This would be applicable to the majority of users.

Prevention advice could be also shown to their physicians with further help to reduce stroke risks. Hypertension, for example can be treated. Paciaroni and Bogousslavsky (2010) suggested that antihypertensive therapy targeted toward the lowest tolerated blood pressure may be beneficial in patients at risk of stroke. Preventive advice given by this system has been supported by past researches. The National Stroke Association and the American Heart Association recommend smoking cessation as a stroke prevention measure (Paciaroni and Bogousslavsky, 2010).

This system helps common users to reduce their stroke risk, however it should never be recommended as a replacement for proper medical treatment. Further developments should also consider calculating uncontrollable variables and unused controllable variables in order to determine stroke risk levels.

\section{REFERENCES}

ASA, 2012. About Stroke. American Heart Association.

Biswas, M.M., S. Sen and J. Simmons, 2009. Etiology and risk factors of ischemic stroke in IndianAmerican patients from a hospital-based registry in New Jersey. USA. Neurol. Asia, 14: 81-86.

Boysen, G. and T. Truelsen, 2000. Prevention of recurrent stroke. Neurol. Sci., 21: 67-72. PMID: 10938183

Bushnell, C.D. and C.S. Colon-Emeric, 2009. Secondary stroke prevention strategies for the oldest patients: Possibilities and challenges. Drugs Aging, 26: 209-230. DOI: 10.2165/00002512-200926030-00003

Duval, B.K. and L. Main, 1994. Expert systems: What is an expert system. Library Software Rev., 13: 44-53.

Ebell, M.H., 2008. Predicting prognoses in patients with acute stroke. Am. Fam. Physician., 77: 1719-1720. PMID: 18619082
Feigin, V.L., 2004. When Lightning Strikes: An Illustrated Guide to Stroke Prevention and Recovery. Kai Tiaki Nursing New Zealand, 10: 23-23.

Hankey, G.J. and C.P. Warlow, 1999. Treatment and secondary prevention of stroke: Evidence, costs and effects on individuals and populations. Lancet, 354 : 1457-1463. PMID: 10543686

Iliffe, S., K. Kharicha, C. Goodman, C. Swift and D. Harari et al., 2005. Smarter working in social and health care (SWISH): Enhancing the quality of life of older people using an 'expert system. Quality Ageing Older Adults, 6: 4-11.

Johnston, S.C., P.M. Rothwell, M.N. Nguyen-Huynh, M.F. Giles and J.S. Elkins et al., 2007. Validation and refinement of scores to predict very early stroke risk after transient ischaemic attack. Lancet, 369: 283-292. PMID: 17258668

Kumar, S. and R.B. Mishra, 2010. Web-based expert systems and services. Knowl. Eng. Rev., 25: 167198. DOI: $10.1017 /$ S0269888910000020

Lynch, G.F., S. Leurgans, R. Raman, A. Barboi and P.B. Gorelick, 2001. A comparison of stroke risk factors in patients enrolled in stroke prevention trials. J. Nat. Med. Associat., 93: 79-86. PMID: 12656439

Martin, J.L. and T.P. Harrison, 1993. Design and implementation of an expert system for controlling health care costs. Operations Res., 41: 819-834. DOI: $10.1287 /$ opre.41.5.819

McCarthy, J., 2007a. What is artificial intelligence? Stanford University.

McCarthy, J., 2007b. What is artificial intelligence? Stanford University.

NHBPEP, 2004. The seventh report of the joint national committee on prevention, detection, evaluation and treatment of high blood pressure. National Institutes of Health.

Paciaroni, M. and J. Bogousslavsky, 2010. Primary and secondary prevention of ischemic stroke. Eur. Neurol., 63: 267-278. DOI: 10.1159/000285183

Panniers, T.L., 2002. Refining clinical terminology for expert system development: An application in the neonatal intensive care unit. Pediatric Nurs., 28: 519-529. PMID: 12424990

Reker, D.M., K. Reid, P.W. Duncan, C. Marshall and D. Cowper et al., 2005. Development of an integrated stroke outcomes database within veterans health administration. J. Rehab. Res. Dev., 42: 77-92. PMID: 15742252 
Sacco, R.L., R. Gan, B. Boden-Albala, I.F. Lin, D.E. Kargman and W.A. Hauser et al., 1998. Leisure-time physical activity and ischemic stroke risk: The Northern Manhattan stroke study. Stroke, 29: 380-387. PMID: 9472878

Sagita, D., 2013. Sounding the Alarm on a Silent Killer: Hypertension. Jakarta Globe.

Schmid, A.A., L. Butterbaugh, C. Egolf, V. Richards and L. Williams, 2008. Prevention of secondary stroke in VA: Role of occupational therapists and physical therapists. J. Rehab. Res. Dev., 45: 1019-1026. PMID: 19165691

Shibata, S., A. Tuchiya, M. Tamura, N. Sasamori and K. Yoshida et al., 1998. Development of a health promotion system for the elderly: Committee of health evaluation for elderly persons council of Japan AMHTS institutions. J. Med. Syst., 22: 43-49. PMID: 9554109
Stockdale, A. and M. Wood, 1992. Building a small expert system for a routine task: A case study. Manage. Decis., 30: 46-49. DOI: 10.1108/00251749210013096

Weir, C.J., G.D. Murray, F.G. Adams, K.W. Muir and D.G. Grosset et al., 1994. Poor accuracy of stroke scoring systems for differential clinical diagnosis of intracranial haemorrhage and infarction. Lancet, 344: 999-1002. PMID: 7934437

WHO, 2006. Global Database on Body Mass Index. World Health Organization.

Zarandi, M.H., M. Zolnoori, M. Moin and $\mathrm{H}$. Heidarnejad, 2010. A fuzzy rule-based expert system for diagnosing asthma. Trans. E: Ind. Eng., 17: $129-142$. 